\title{
Total and functional parasite specific IgE responses in Plasmodium falciparum-infected patients exhibiting different clinical status Joana Duarte ${ }^{1}$, Prakash Deshpande ${ }^{2}$, Vincent Guiyedi ${ }^{3,4}$, Salah Mécheri ${ }^{5}$, Constantin Fesel ${ }^{1}$, Pierre-André Cazenave ${ }^{3}$, Gyan C Mishra², Maryvonne Kombila ${ }^{4}$ and Sylviane Pied*1,3
}

\begin{abstract}
Address: ${ }^{1}$ Instituto Gulbenkian de Ciencia, LEA CNRS-IGC, Oeiras, Portugal, ${ }^{2}$ National Centre for Cell Sciences, Pune, India, ${ }^{3}$ Unité d'Immunophysiopathologie Infectieuse, Institut Pasteur, 25 rue du Docteur Roux, 75 724, Paris Cedex 15, France, ${ }^{4}$ Département de ParasitologieMycologie-Médecine Tropicale, Faculté de Médecine de Libreville, Gabon and ${ }^{5}$ Unité des Réponses Immunes Précoces aux Parasites, Institut Pasteur Paris, France

Email: Joana Duarte - jduarte@igc.gulbenkian.pt; Prakash Deshpande - pdeshpande@nccs.in; Vincent Guiyedi - guidivin@pasteur.fr; Salah Mécheri - mecheri@pasteur.fr; Constantin Fesel - cfesel@igc.gulbenkian.pt; Pierre-André Cazenave - cazenave@pasteur.fr; Gyan C Mishra - gcishra@nccs.in; Maryvonne Kombila - valentine_favry@yahoo.fr; Sylviane Pied* - spied@pasteur.fr

* Corresponding author
\end{abstract}

Published: 04 January 2007

Malaria Journal 2007, 6:1 doi:10.1186/1475-2875-6-1

This article is available from: http://www.malariajournal.com/content/6/I/I

(C) 2007 Duarte et al; licensee BioMed Central Ltd.

This is an Open Access article distributed under the terms of the Creative Commons Attribution License (http://creativecommons.org/licenses/by/2.0), which permits unrestricted use, distribution, and reproduction in any medium, provided the original work is properly cited.
Received: 25 October 2006

Accepted: 04 January 2007

\begin{abstract}
Background: There is an increase of serum levels of lgE during Plasmodium falciparum infections in individuals living in endemic areas. These lgEs either protect against malaria or increase malaria pathogenesis. To get an insight into the exact role played by $\lg E$ in the outcome of $P$. falciparum infection, total IgE levels and functional anti-parasite IgE response were studied in children and adults, from two different endemic areas Gabon and India, exhibiting either uncomplicated malaria, severe non cerebral malaria or cerebral malaria, in comparison with control individuals.

Methodology and results: Blood samples were collected from controls and P. falciparum-infected patients before treatment on the day of hospitalization (day 0 ) in India and, in addition, on days 7 and 30 after treatment in Gabon. Total IgE levels were determined by ELISA and functional P. falciparum-specific IgE were estimated using a mast cell line RBL-2H3 transfected with a human Fce RI $\alpha$-chain that triggers degranulation upon human IgE cross-linking. Mann Whitney and Kruskall Wallis tests were used to compare groups and the Spearman test was used for correlations.

Total lgE levels were confirmed to increase upon infection and differ with level of transmission and age but were not directly related to the disease phenotype. All studied groups exhibited functional parasitespecific IgEs able to induce mast cell degranulation in vitro in the presence of $P$. falciparum antigens. Plasma IgE levels correlated with those of IL- 10 in uncomplicated malaria patients from Gabon. In Indian patients, plasma IFN- $\gamma$, TNF and IL-I0 levels were significantly correlated with IgE concentrations in all groups.

Conclusion: Circulating levels of total lgE do not appear to correlate with protection or pathology, or with anti-inflammatory cytokine pattern bias during malaria. On the contrary, the $P$. falciparum-specific lgE response seems to contribute to the control of parasites, since functional activity was higher in asymptomatic and uncomplicated malaria patients than in severe or cerebral malaria groups.
\end{abstract}




\section{Background}

Malaria is a complex disease that kills between one and two million people every year. Most of those affected are children under five years of age, non-immune individuals and pregnant women [1]. The principal cause of death is infection by Plasmodium falciparum due to its ability to induce severe complications such as severe anaemia and/ or cerebral malaria (CM) often associated with hypoglycaemia [2-4]. The physiopathology of malaria cannot be represented by a single scheme. For example, patients who develop CM present a range of acute neurological manifestations and the disease is characterized by a diffuse encephalopathy, altered levels of consciousness, deep coma and seizure leading to death. Even though during the last few years a lot of information has become available from clinical and experimental studies, the causes of $\mathrm{CM}$ remain to be determined. The clinical outcome of a $P$. falciparum infection depends on the genetic factors of the host and parasite, and also on host immune responses. Antibodies and $\mathrm{T}$ cells are among the immune factors thought to play a role in mediating protection and also pathology [2-5].

P. falciparum infection increases the serum levels of IgM and IgG antibodies but also IgE in individuals living in endemic areas [6-12]. IgEs may protect against or participate in malaria pathogenesis. The association of high anti$P$. falciparum IgE levels with a reduced risk of developing clinical malaria suggests the involvement of IgE in protection $[13,14]$. The observation that circulating levels of IgE most often correlate with severe rather than uncomplicated disease suggests a pathogenic role of $\operatorname{IgE}[8,10-12]$, and the positive correlation between the levels of $\operatorname{IgE} / \mathrm{IgE}$ immune complexes and the levels of TNF in CM patients provides supporting evidence [8,10-12]. The exact role played by IgE in malaria is still unclear.

IgE is an immunoglobulin isotype that only exists in mammals. It is present at very low concentrations in the serum of normal individuals, at levels ranging from 10 to $300 \mathrm{ng} / \mathrm{ml}$ [9]. Its functional effect has been shown to depends on Fc receptors expressed on mast cells and basophils both in mice and humans, as well as on eosinophils, monocytes/macrophages and platelets in humans [9]. IgEs positively regulate both of their receptors: the high affinity receptor (Fce RI) and the low affinity receptor (FCE RII or CD23) [15]. The FCE RI is expressed only on mast cells and/or basophils in both mice and humans $[9,16]$. The binding of IgE to the high affinity receptor on the mast cell membrane and its subsequent aggregation with antigens results in degranulation and the release of mediators that further aggravate an ongoing allergic process [17]. On basophils, the cross-linking of Fce RI-bound IgE rapidly induces the release of IL- 4 and IL-13 [16], among other inflammatory mediators. The low affinity receptor (Fce RII) is the second major and widely distributed IgE receptor. It is also known as CD23 and is constitutively expressed on B cells and is induced by IL-4 on macrophages, some T cells, human eosinophils and platelets $[9,16]$. The cross-linking of CD23 on macrophages or on other CD23-bearing effector cells by IgEcontaining immune complexes is thought to play a pathogenic role in malaria via TNF-mediated pathways [16].

This study aimed to evaluate the total and functional $P$. falciparum-specific IgE responses, the association of these responses with plasma cytokine patterns and the phenotype of the disease in endemic controls and infected patients with different clinical forms of malaria. The infected patients originated from a low endemic area in India and a high endemic area in Gabon.

\section{Materials and methods Study population}

Patients from Gabon

All the patients included in this study were children aged between 0.1 and 6 years (mean age $=2.6$ years) recruited between 1996 and 2000 at the Owendo Pediatric Hospital (OPH) and the Libreville Hospital Center (LHC) in Gabon (see Table 1). Informed parental consent had been obtained. Gabon has an equatorial climate that is hot and humid, with an endemic malaria transmission. The study design was approved by the local health office ethics committee. The patients were distributed into different groups according to World Health Organization (WHO) guidelines for the definition of uncomplicated and severe malaria [18]. A cohort of 135 P. falciparum-infected children was constituted and divided into three groups according to disease severity [[6], 67] 50patients with uncomplicated malaria (UM), 29 with severe non-cerebral malaria (SM) developing severe anaemia (haemoglobin level $<5 \mathrm{~g} / \mathrm{dl}$ ), or hypoglycaemia (glycaemia $<2.2$ $\mathrm{mmol} / \mathrm{ml}$ ), and 17 with severe cerebral malaria (CM) with a Blantyre Coma Score $<2$, or three convulsive episodes during 24 hours before admission with post-critical comatose > 15 minutes]. Two control groups were recruited: an uninfected group, also called endemic control (EC) group, comprising 17 children with $P$. falciparum-negative thin blood smear, and asymptomatic infected group (AI) comprising 22 children with no clinical manifestation of malaria but a $P$. falciparum-positive thin blood smear.

\section{Patients from India}

Malaria patients were recruited in the village of Gondia, an endemic region in the north-east of the Maharashtra State of India. The village is surrounded by forest. Gondia has been known as an endemic area for at least the last 20 years. P. falciparum appeared in Gondia over the last 10 years. It is transmitted during the rainy season in June, 
Table I: Characteristics of both studied cohorts: Gabon and India. Clinical group description, according to the number of patients, age, sex and parasitaemia.

\begin{tabular}{|c|c|c|c|c|c|}
\hline & Clinical Groups & Staff & Age-mean (min-max) & Sex (male-female) & Parasitemia (\%) \\
\hline \multirow[t]{6}{*}{ Gabon } & EC & 17 & $2,7(0,5-5)$ & $10-7$ & - \\
\hline & Al & 22 & $2,9(0,1-5)$ & $12-10$ & $0.22(0.0 \mathrm{I}-1.2)$ \\
\hline & UM & 50 & $3(0,5-5)$ & $22-28$ & $6.88(0.05-48.6)$ \\
\hline & SM & 29 & $\mathrm{I}, 8(0,2-4,5)$ & $|8-1|$ & $5.91(0.08-31)$ \\
\hline & $\mathbf{C M}$ & 17 & $2,4(0,5-5)$ & $12-5$ & $10.42(0.15-64)$ \\
\hline & Total & 145 & $2,6(0,1-5)$ & $74-61$ & \\
\hline \multirow[t]{7}{*}{ Índia } & NEC & 9 & $32,7(25-63)$ & $8-1$ & - \\
\hline & EC & 14 & $27,3(23-37)$ & $13-1$ & - \\
\hline & UM & 31 & $30,8(4-70)$ & $18-13$ & $\mathrm{I}, 24(0,7 \mathrm{I}-2,18)$ \\
\hline & SM & 13 & $30,4(8-65)$ & $10-3$ & $\mathrm{I}, \mathrm{II}(0,38-3,2 \mathrm{I})$ \\
\hline & CM & 26 & $40,4(9-72)$ & $16-10$ & $2,04(1,07-3,87)$ \\
\hline & ExCM & 5 & $19,36(7-51)$ & $0-5$ & - \\
\hline & Total & 93 & $32,3(4-72)$ & $65-28$ & $1,50(1,02-2,20)$ \\
\hline
\end{tabular}

NEC- non endemic control, EC- endemic control, Al- asymptomatic infected, UM - uncomplicated malaria, SM - severe malaria (non-cerebral), CM - cerebral malaria, ExCM - Ex-cerebral malaria. Note: sex couldn't be determined for I/I8, II/6I and 4/33 patients from EC, UM and SM, respectively.

peaks in the winter season (November, December and January) and becomes rare as summer approaches (March, April and May). The studied groups consisted of 98 patients from four to 72 years of age, being predominantly adults (see Table 1). Six cohorts were constituted according to WHO criteria for uncomplicated and severe malaria: two control groups of uninfected individuals from non-endemic (NEC) and endemic regions (EC) comprising nine and 14 patients respectively; three groups of infected patients, with 31 developing uncomplicated malaria (UM), 13 developing severe non-cerebral malaria (SM) and 26 developing cerebral malaria (CM); and one group of five patients that had recovered from CM (ex-cerebral malaria patients, Ex-CM). Between eight and 10\% of the CM malaria cases died. UM cases were treated as outpatients. SM patients were admitted to hospital fully conscious and could respond well verbally to doctors' questions. CM cases were in coma. Drug treatment was paracetamol, quinine and arteether $\left(\mathrm{E}-\mathrm{mal}{ }^{\circledR}\right)$. Samples were collected after obtaining the consent of the patients, or of their families. Blood samples from endemic controls were collected from the relatives of malaria patients (brothers/sisters/parents) with their consent. These controls had not suffered from malaria during the previous two years. Non-endemic blood samples were collected from individuals who had not suffered from malaria during the previous five years.

\section{Blood sample collection and parasite assessment}

Venous blood was collected on EDTA in sterile vacutainers from each patient on the day of hospitalization (day 0 , before any treatment), and seven (day 7) and thirty days later (day 30). Plasma was obtained by centrifuging the blood samples at $5000 \mathrm{rpm}$ for $15 \mathrm{~min}$. Plasma samples were stored at $-80^{\circ} \mathrm{C}$ until use.

Parasitaemia was assessed by counting asexual forms of $P$. falciparum on thin blood smears under a light microscope after Giemsa staining. Parasitaemia was expressed as the mean percentage of infected red blood cells.

\section{Culture of malaria parasites}

Erythrocytic stages of the P. falciparum malaria parasite line FAN 5HS (source: NCCS, Pune, India) and 3D7 were cultured using candle jar dessicators as previously described [19]. The culture medium was RPMI 1640 (Gibco-BRL), supplemented with 0.5\% AlbuMix (Gibco $\mathrm{BRL}$ ). The cultures were maintained in six-well or 24-well tissue culture plates (NUNC). Parasitaemia was $5 \%$ at the start of culture and reached $25 \%$ after six days. Culture medium and fresh RBCs were added every other day.

\section{Preparation of parasite extracts}

Parasite soluble antigen was prepared from synchronous cultures containing more than $20 \%$ mature trophozoites; more than $6 \%$ rings and more than $5 \%$ schizonts were used. The cultures were pooled and centrifuged at 3,000 rpm at $4{ }^{\circ} \mathrm{C}$, and the pRBC pellet was kept and the supernatant discarded. The pRBC pellet was suspended in 10 $\mathrm{ml}$ sterile PBS $1 \times(0.15 \mathrm{M}, \mathrm{pH} 7.2)$ and then centrifuged. The parasitized red blood cell (pRBC) pellet was washed five times and then lysed by adding $15 \mathrm{ml}$ of $0.1 \%$ saponin. The saponin treatment frees the parasites from the infected RBCs. This was centrifuged at 6,000 rpm for $30 \mathrm{~min}$ at $4^{\circ} \mathrm{C}$. The supernatant was discarded and the parasite pellet was washed five or six times with sterile 
cold PBS. The parasite pellet was resuspended in $1 \mathrm{ml}$ protein isolation buffer containing a cocktail of protease inhibitors. This was briefly sonicated and the tube was kept at $4{ }^{\circ} \mathrm{C}$ for between four and five hours. The contents of the tube were agitated by cyclo-mixing and then centrifuged at $6,000 \mathrm{rpm}$ for $30 \mathrm{~min}$ at $4^{\circ} \mathrm{C}$. The clean supernatant was collected in a separate tube and the pellet was discarded. The contents were sterilized by passing through $0.22 \mu \mathrm{m}$-pore filters. Aliquots of the antigen were frozen at $-70^{\circ} \mathrm{C}$ until use. Parasite proteins were quantified by the Bradford method. The concentration of the parasite line FAN 5HS and 3D7 were 1.2 and $2.6 \mathrm{mg} / \mathrm{ml}$, respectively.

\section{Normal RBC extracts}

Normal red blood cell (RBC) extract was prepared from the same batch of RBCs used for culturing the parasites, and followed the same procedure as previously described for pRBCs. Briefly, the RBCs were washed with PBS and the buffy coat was removed. After centrifugation, the RBC pellet was suspended in $1 \mathrm{ml}$ protein isolation buffer containing a cocktail of protease inhibitors. This was briefly sonicated and the tube was kept at $4^{\circ} \mathrm{C}$ for between four and five hours. The contents of the tube were agitated by cyclo-mixing and then centrifuged at $6000 \mathrm{rpm}$ for $30 \mathrm{~min}$ at $4{ }^{\circ} \mathrm{C}$. The clean supernatant was collected in a separate tube and the pellet discarded. The protein contents were estimated using a protein determination kit (BCATM protein assay Kit, Pierce, France).

\section{Total lgE levels}

An ELISA method was used to detect total IgE plasma levels in samples corresponding to day 0 , day 7 and day 30 . ELISA plates (96 microwell plates, reacti-bind 96 EIA Plate 100/PKG, Pierce) were coated with $50 \mu \mathrm{l} /$ well of purified sheep polyclonal anti-human IgE solution at $5 \mu \mathrm{g} / \mathrm{ml}$ (The Binding Site, Birmingham UK) by incubation overnight at $4^{\circ} \mathrm{C}$. The plasma samples were diluted 1:5 and incubated for two hours at $37^{\circ} \mathrm{C}$. Bound IgE was detected using a peroxydase-conjugated polyclonal anti-human-IgE (The Binding Site, Birmingham UK). Binding was revealed using the OPD substrate (Sigma) and the product was quantified from the optical density (OD) at $450 \mathrm{~nm}$. Serial dilutions ranging from $2 \mu \mathrm{g} / \mathrm{ml}$ to $0.0019 \mu \mathrm{g} / \mathrm{ml}$ of IgE solution (human monoclonal IgE provided by Dr Thierry Batard - Stallergenes, Anthony, France) gave the standard curve. The median of each optical density value was fitted into the sigmoidal standard curve using a specific ELISA programme running in Igor version 3.16 (Wavemetrics, Lake Oswego, OR).

\section{IgE functional assay}

A new rat mast cell line RBL-2H3 transfected with a human Fce RI $\alpha$-chain that triggers degranulation upon human IgE cross-linking was used [20]. Cells were main- tained in Dulbecco medium (Gibco BRL, Eragny, France) containing $10 \%$ foetal bovine serum (FCS), $100 \mathrm{U} / \mathrm{ml}$ penicillin and $100 \mathrm{U} / \mathrm{ml}$ streptomycin (GIBCO BRL, France). Cells were expanded by incubation at $37^{\circ} \mathrm{C}$ for three to four days in complete Dulbecco medium supplemented with G418 (GIBCO BRL, France).

$\beta$-Hexosaminidase is known as a component of the basophil and the mast cell specific granule, and is released during degranulation of these cells [21]. Degranulation was monitored after antigen stimulation by measuring the level of released $\beta$-hexosaminidase. Fce RI $\alpha$-chain RBL$2 \mathrm{H} 3$ transfected rat mast cell line cultures $\left(5 \times 10^{5}\right.$ cells per well) were incubated with the different serum samples at a non-cytotoxic dilution (previously determined) for 48 hours at $37^{\circ} \mathrm{C}$ in the absence of the G418 antibiotic. The upregulated receptors were saturated by incubation at $4{ }^{\circ} \mathrm{C}$ for 30 minutes with the same samples diluted 1:10. The cells were then washed with PBS $1 \mathrm{X}$, centrifuged and resuspended in $1 \mathrm{ml}$ Tyrode buffer before being centrifuged again. Finally, the cell pellet was resuspended in $450 \mu \mathrm{l}$ of D2O (50\%) and Tyrode buffer (50\%) solution and each culture sample was distributed to 10 ELISA plate wells. Different controls were carried out for each sample. Control cells on lane 1 and 2 were subjected to Triton disruption (Triton 5\%) and represented 100\% enzyme release. Cells on lanes 3 and 4 were incubated with $50 \mu \mathrm{l}$ of complemented Dulbecco medium without serum and represented the background enzyme release. Lanes 5 and 6,7 and 8,9 and 10 were incubated with $50 \mu \mathrm{l}$ of different duplicated concentrations of parasite extract $(1,000,100$ and $10 \mathrm{ng} / \mathrm{ml}$ ) for 30 minutes at $37^{\circ} \mathrm{C}$. After centrifugation of each well sample, $50 \mu \mathrm{l}$ of each supernatant was collected and incubated with $50 \mu \mathrm{l}$ of PNAG substrate solution for 90 minutes at $37^{\circ} \mathrm{C}$. The level of released $\beta$ hexosaminidase was estimated from the OD at $405 \mathrm{~nm}$ using a spectrophotometer. All results are expressed as the percentage of total $\beta$-hexosaminidase in the cells after correcting for spontaneous release in unstimulated cultures, calculated as following: (experimental $\beta$-hexosaminidase - background $\beta$-hexosaminidase)/(total $\beta$-hexosaminidase - background $\beta$-hexosaminidase $) \times 100$.

\section{Flow cytofluorometry analysis}

FACS analysis was performed after incubating RBL-2H3D12.8 cells with several dilutions of serum samples to follow the induction of the high affinity receptor (Fce RI) expression after stimulation by IgEs in the patient's sera. Cells were incubated for 48 hours at $37^{\circ} \mathrm{C}$ with the different serum samples optimally diluted to avoid cytotoxicity. A saturation step with the same sera diluted 1:10 was done by incubation at $4^{\circ} \mathrm{C}$ for 30 minutes. Cells were washed with PBS $1 \mathrm{X}$ and incubated with FITC-labelled anti-IgE (Tebu, Le Perray en Yvelines, France) (1/100) for 30 minutes. Cells were washed again, centrifuged, resuspended in 
PBS 1X and analysed by cytofluorometry using Cellquest software (Beckton Dickinson, USA). 10,000 cells were acquired per tube.

\section{Cytokine levels}

The levels of cytokines in the plasma (IL-4, TNF, INF- $\gamma$, and IL-10) were estimated by Opti-ELISA kits (Pharmingen, San Diego, CA.USA) used following the manufacturer's instructions.

\section{Statistics}

Due to a non-normal distribution of the scores in each group, non-parametric tests were performed, using the median to compare the different clinical groups. The Mann Whitney test was used for comparisons between two groups and the Kruskall Wallis test to compare three or more groups. Spearman's correlation was used to check for correlations between parameters. P values less than 0.05 were considered as significant. Chi-squared test was used to compare qualitative variables.

\section{Results}

Serum total IgE levels in groups of P. falciparum infected patients with different clinical phenotypes

Total IgE levels were analysed in endemic controls and in cohorts of $P$. falciparum-infected patients with different clinical forms of malaria, ranging from asymptomatic to cerebral disease, from Gabonese and Indian endemic areas to study the association between the $\operatorname{IgE}$ response and disease severity. Total IgE levels were measured by ELISA in individual sera before drug administration (corresponding to day 0 ) and determined the general distribution in the studied populations from Gabon (Figure 1A) and India (Figure 1B). Total IgE concentrations were found to be much higher in patients from India (mainly adults) than in patients from Gabon (children). In both populations independent of the different levels of $\operatorname{IgE}$ in each population, the median IgE levels within each clinical group tended to increase upon infection (mainly in UM and SM groups), although the difference between the groups was only significant in the Indian population (Kruskall Wallis, $\mathrm{p}=0.0005)$. As only Indian patients showed a significant difference, the Mann Whitney test was used to compare the different groups in this population only. There was a significant increase in IgE levels in the EC group compared to the NEC group $(p=0.042)$. The most significant increase in IgE levels (versus the EC group) occurred in the UM patients $(\mathrm{p}=0.015)$ and in the SM patients $(\mathrm{p}=0.013)$. No significant difference between the EC group and the CM and Ex-CM groups was observed.

A range of values of IgE levels was defined enabling the analysis of the frequency of normal, moderate and high IgE levels in each clinical group of patients. The so-called normal values were adjusted to the studied population because the Gabonese and Indian groups had different plasma total IgE ranges. Therefore, the normal value $(\mathrm{N})$ was defined by the median IgE levels in the endemic controls of each study population. Consequently, all values between $\mathrm{N}$ and $2 \mathrm{~N}$ were considered as low/moderate IgE levels and those between $2 \mathrm{~N}$ and $3 \mathrm{~N}$ as moderate/high IgE levels, with the highest levels being above $3 \mathrm{~N}$ (Figures $1 \mathrm{C}$ and 1D). Even in Gabonese patients, for whom the increase of IgE in the disease groups was not significant, a higher percentage of patients with clinical disease had higher IgE levels than controls and asymptomatic patients. These differences were more marked in the UM, $\mathrm{SM}$ and $\mathrm{CM}$ Indian patients (Figure 1D). In the Indian population, the NEC group did not have moderate/high IgE levels, although a high percentage of patients exhibited normal IgE levels (Figure 1D). Also, no significant change was detected in IgE levels over time in the UM, SM and CM groups of the Gabonese cohorts when tested seven days and 30 days after treatment (Table 2). No significant association of malaria and IgE levels with sex in the two studied populations. However, a significant increase in IgE levels with age $(\mathrm{p}=0.00034)$ was observed in the Gabonese subjects (Figure 2A) but not in the Indian subjects.

The correlation between IgE levels and the parasite load was tested. Although the general trend was different in the Indian and Gabonese population, there was no significant correlation between IgE concentration and parasite load for all groups together. In Gabonese cohorts, a negative correlation for all groups was observed, except for the UM patients where the correlation showed a positive tendency. In the Indian cohorts, a positive correlation was observed between IgE levels and parasite load, mainly in the UM and SM groups (Figure 2B).

\section{Functional parasite specific IgE response in $\mathbf{P}$. falciparum infected patients}

Previous studies have used ELISA to quantify specific IgE present in the serum $[7,8,10,11]$. The functionality of specific IgEs present in the serum was studied by evaluating the ability of these IgEs to induce mast cell degranulation in the presence of the parasite antigen. A rat mast cell line transfected with the human $\alpha$-chain of FcE RI was used [20]. Human FcE RI expression was induced after incubation with all serum samples at non-cytotoxic dilutions. FACS was used to detect the presence of FcåRI receptors on the mast cells surface induced by IgE present in serum samples. Although the fluorescence intensity revealing human Fce RI expression by the mast cells varied between patient samples, IgE receptors were upregulated in all the samples tested. No correlation between total IgE levels in the serum and the up-regulation of mast cell receptors was found. There was no significant correlation between IgE 
A

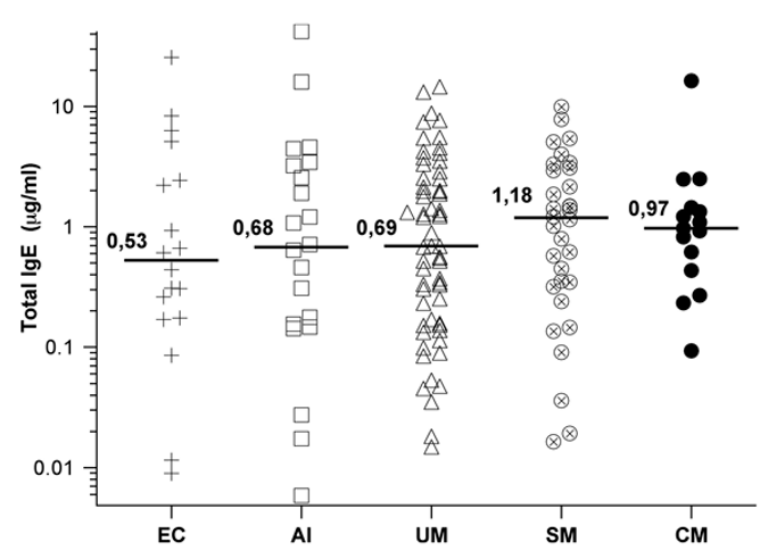

C

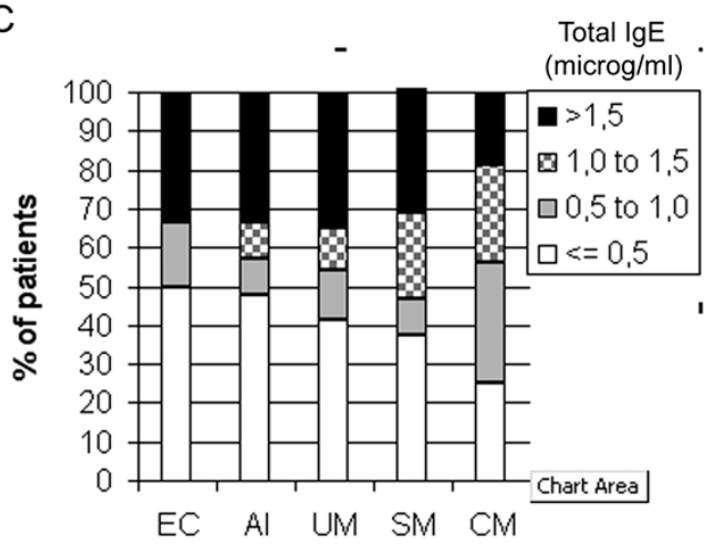

B
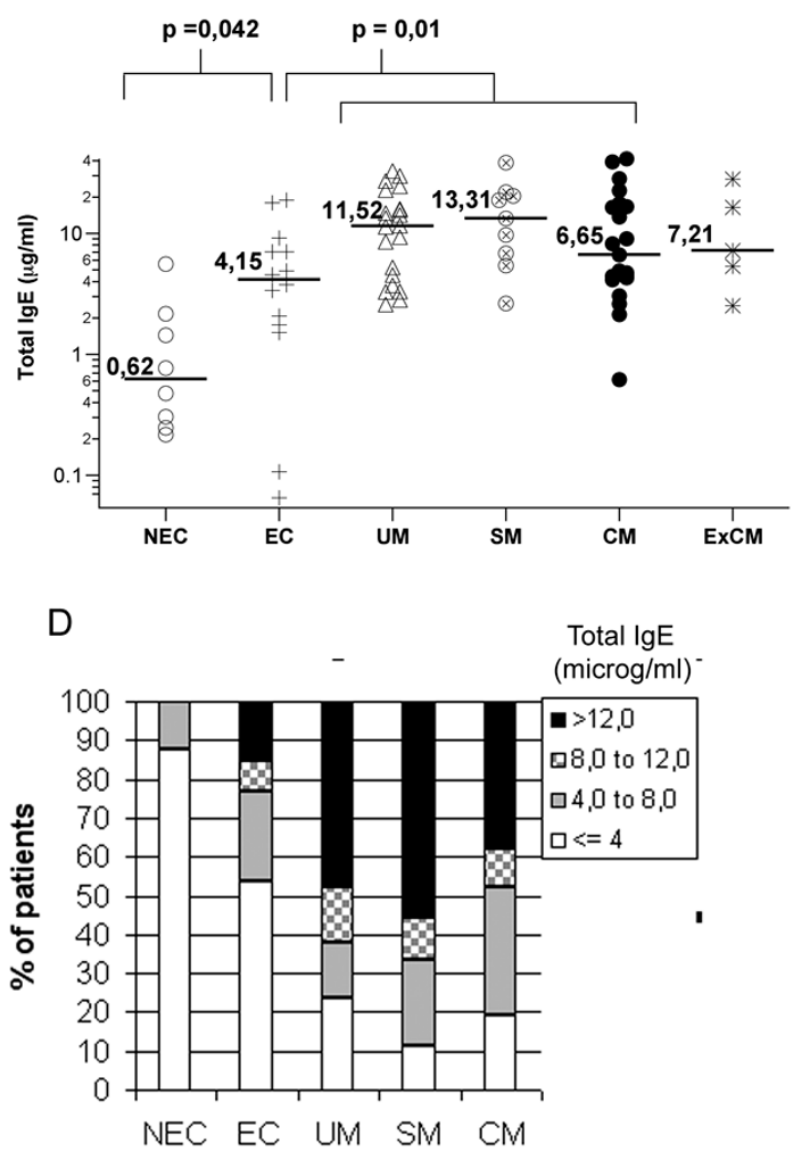

Figure I

Distribution of total lgE levels per clinical group in both studied populations: Gabon and India. A. Total lgE levels ( $\mu \mathrm{g} / \mathrm{ml})$ per clinical group in Gabonese patients (non-significant Kruskall Wallis test). B. Total lgE levels $(\mu \mathrm{g} / \mathrm{ml})$ in Indian patients (significant Kruskall Wallis test, $P=0.0005$ ). C. Percentage of patients with defined IgE levels per group in the Gabonese population (normal levels $(\mathrm{N})$ lower than or equal to $0.500 \mu \mathrm{g} / \mathrm{ml}$, moderate levels $(\mathrm{N}$ to $2 \mathrm{~N})$, from $0.50 \mathrm{I}$ to $1.000 \mu \mathrm{g} / \mathrm{ml}$, high levels $(2 \mathrm{~N}$ to $3 \mathrm{~N})$, from I,000 to I,500 $\mu \mathrm{g} / \mathrm{ml}$, very high ( $>3 \mathrm{~N})$ greater than I,500 $\mu \mathrm{g} / \mathrm{ml})$. D. Percentage of patients with defined levels of lgE per group in the Indian population (normal levels $(N)$ lower than or equal to $4,000 \mu \mathrm{g} / \mathrm{ml}$, moderate levels $(N$ to $2 N$ ), from $4,000$ to $8,000 \mu \mathrm{g} / \mathrm{ml}$, high levels ( $2 \mathrm{~N}$ to $3 \mathrm{~N}$ ), from 8,000 to $12,000 \mu \mathrm{g} / \mathrm{ml}$, very high $(>3 \mathrm{~N})$, greater than $12,000 \mu \mathrm{g} / \mathrm{ml})$. Legend: $\mathrm{EC}$ - endemic control, Al - Asymptomatic infected, UM - uncomplicated malaria, SM - severe malaria, CM - cerebral malaria, NEC - non-endemic control, ExCM - ex-cerebral malaria.

levels and receptor upregulation and the level of mast cell degranulation. Sensitized cells were then incubated with different concentrations of a $P$. falciparum blood-stage antigen extract. Mast cell degranulation was measured by quantifying the release of $\beta$-hexosaminidase. Control cells not exposed to any serum gave a maximum mast cell degranulation of $3 \%$. Therefore, serum samples giving an enzyme release greater than $5 \%$ in the presence of at least one of the antigen concentrations were considered positive for functional IgE. In the Gabonese cohorts (Figure 3A), there were functional $P$. falciparum IgE in all clinical groups. However, the percentage of patients with functional specific anti-parasite IgE was higher in asymptomatic and uncomplicated malaria patients than in other groups. Also, the percentage of patients displaying parasite-specific IgE was lower in the group exhibiting severe disease. The distribution of patients per group releasing between 5 and $10 \%, 10$ and $30 \%$ and above 30\% $\beta$-hexosaminidase induced by specific anti-parasite IgE revealed that one patient in CM group had a degranulation level above $30 \%$, being the highest induced response among all the tested individuals. The same assay was carried out on 
Table 2: Day 0, day 7 and day 30 median IgE levels per clinical group in the Gabonese population.

\begin{tabular}{cccccc}
\hline Total IgE $(\mu \mathrm{g} / \mathrm{ml})$ & EC (min-max) & Al (min-max) & UM (min-max) & SM (min-max) & CM (min-max) \\
\hline Day 0 & $0,525(0,009-25,47)$ & $0,677(0-41,96)$ & $0,690(0,015-13,173)$ & $1,132(0,016-9,915)$ & $0,922(0,093-16,34)$ \\
Day 7 & $0,582(0,0144-5,644)$ & $0,448(0,0192-3,188)$ & $0,516(0,011-5,228)$ & $0,836(0,016-5,689)$ & $8,033(8,033-8,033)$ \\
Day 30 & $1,026(0,044-2,628)$ & $0,977(0,056-7,446)$ & $0,151(0,052-3,925)$ & $0,662(0,011-6,315)$ & ---- \\
\hline
\end{tabular}

EC - endemic control, Al - asymptomatic infected, UM - uncomplicated malaria, SM - severe malaria (non-cerebral), CM - cerebral malaria.

the Indian population. Although there was no significant difference between groups, the percentage of patients having functional IgE recognizing the parasite extract was slightly higher in EC and UM groups than in SM and CM groups (Figure 3B). All positive patients had an enzyme release of between 10 and 30\%. No significant correlation was found between $P$. falciparum-specific IgE-induced mast cell degranulation levels and sex, age and parasitaemia.

\section{Relationship between total and specific serum IgE and cytokine profiles}

IgE production is influenced by cytokines produced by activated $\mathrm{T}$ cells. These cytokines are also involved in pathophysiological mechanisms associated with severe malaria $[4,22,23]$. Therefore, the relationship between the cytokine profile, the IgE levels and the clinical manifestation was investigated. IFN- $\gamma$, TNF and IL-10 levels were measured in the sera of the Indian and Gabonese patients. IL-4 levels were measured only in Indian patients as it was not different between the Gabonese groups. IFN $\gamma$ concen- trations were highest in the Gabonese AI and CM groups (Table 3). This cytokine is significantly higher in the AI and $\mathrm{CM}$ groups than in $\mathrm{EC}(\mathrm{p}=0.02$ and $\mathrm{p}=0.009$ respectively). The plasma TNF concentration was similar in the severe SM and CM groups and in disease-free EC and AI groups. TNF levels were clearly higher in SM and CM groups than in the UM group $(\mathrm{p}<0.001)$. Surprisingly, EC and $\mathrm{AI}$ also exhibited higher TNF levels when compared to UM group. No association between IFN- $\gamma$ or TNF and IgE levels were found. However, a significant positive correlation was found between the concentration of total $\operatorname{IgE}$ and IL-10 in the UM group ( $\mathrm{p}=0.02)$ and a significant negative correlation in the AI group $(\mathrm{p}=0.02)$ (Figure 4$)$. The median levels of the different cytokines in the plasma of Indian patients are given in Table 4. IL-10 and TNF levels were higher in CM patients than in controls and other $P$. falciparum-infected patients. The plasma concentrations of these cytokines were moderate in cured $\mathrm{CM}$ patients (ExCM). Their levels of IL-10 and TNF were slightly higher in endemic controls than in non-endemic controls. Levels of IFN- were lower in the CM group than in AI group. No
A

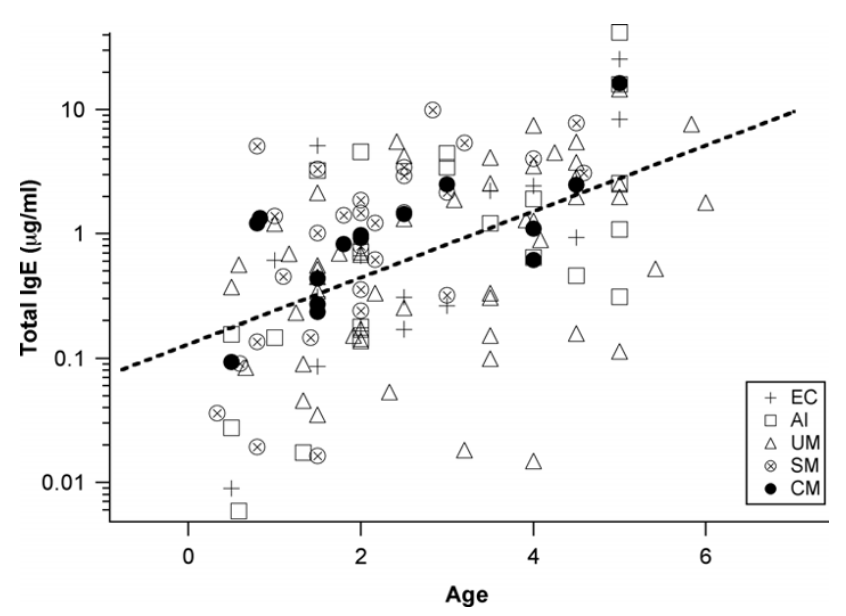

B

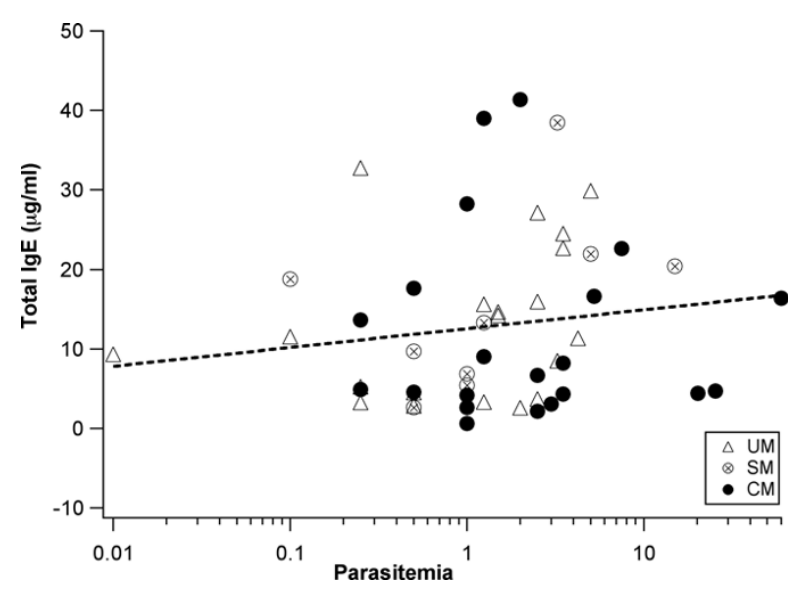

\section{Figure 2}

A. Total IgE correlation with age in the Gabonese population (significant spearman correlation, $\mathrm{P}=\mathrm{I} .0 \times 10-9)$. B. Total IgE correlation with parasitaemia in the Indian population. Significant Spearman correlation $(p=0,000 \mathrm{I})$. 

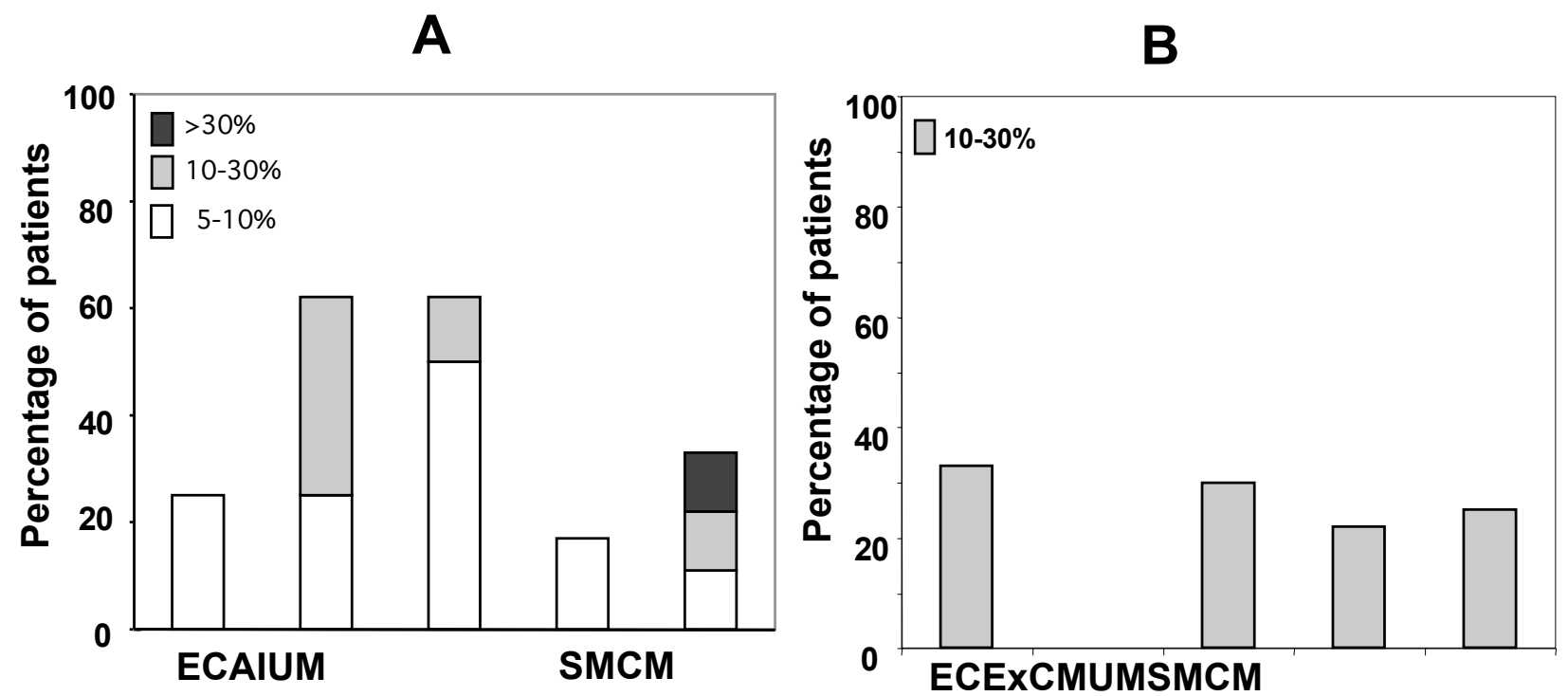

Figure 3

Percentage of patients with positive functional IgE against parasite antigen in the Gabonese and Indian populations. A. Distribution of patients with positive anti-parasite functional lgE, exhibiting different intensities of enzyme release per clinical group in the Gabonese population (low enzyme release, from 5 to 10\%; moderate enzyme release, from 10 to $30 \%$; and high enzyme release, greater than than $30 \%$ ). B. Distribution of patients with positive anti-parasite functional IgE in the Indian population.

difference was found for the levels of IFN- $\gamma$ between the uncomplicated and severe disease. Although, there was a significant correlation between IgE levels and IFN- $\gamma$ (Figure 5A), TNF (Figure 5B) and IL-10 (Figure 5C) levels when looking at all the groups combined. Most diseased groups had high cytokine levels, whereas control groups had lower levels (Figure 5A, B and 5C).

\section{Discussion}

The main feature of this study is the comparison of total and functional $P$. falciparum-specific IgE responses in two populations of low and high malaria transmission levels, India and Gabon respectively and their relationship with disease severity. Several clinical groups were compared: endemic non-infected controls, asymptomatics and different clinical manifestations including uncomplicated, severe non-cerebral and cerebral malaria.
Indian and Gabonese individuals exhibited a different range of plasma levels of circulating IgE These cohorts had a different age range, with the Gabonese groups being children from 0 to 6 years of age, whereas Indian patients had a mean age of 30 years. In the two populations, irrespective of its concentration and also consistent with previously published data, the IgE distribution tended to increase upon parasite stimulation $[8,10,11]$. In the Indian population, circulating IgE levels were seven times higher in endemic controls than in non-endemic controls. This suggests that exposure to the parasite strongly influences the production of IgE, although this difference may also be due to other endemic factors [7]. Nevertheless, it was reported that IgE levels were greatest in patients developing severe disease than in CM group (Figure 1A and $1 \mathrm{~B})$. When considering the percentage of patients that produce high levels of IgE per group, it was shown that IgE

Table 3: Cytokine distribution in the Gabonese population: median TNF, IFN- $\gamma$ and IL- 10 levels per clinical group.

\begin{tabular}{|c|c|c|c|c|c|}
\hline & EC (min-max) & Al (min-max) & UM (min-max) & SM (min-max) & CM (min-max) \\
\hline TNF (pg/ml) & $4 I$ (8-94) & $36(0-395)$ & $8(0-440)$ & $175(I-442)$ & $209(0-1520)$ \\
\hline IFN- $\gamma$ (pg/ml) & $3,2(0-8)$ & $9,5(0-31)$ & $5(0-395)$ & $4(0-20 I)$ & $6,2(2,5-9)$ \\
\hline IL-I 0 (pg/ml) & $14(0-83)$ & $102(0-3 \mid 7)$ & $134(0-1380)$ & $339(0-5200)$ & $95(0-2300)$ \\
\hline
\end{tabular}

EC - endemic control, Al - asymptomatic infected, UM - uncomplicated malaria, SM - severe malaria (non-cerebral), CM - cerebral malaria. Comparing group by group with the Endemic control: TNF was significantly lower in UM ( $p=0,0006)$; IFN- $\gamma$ was significantly higher in Al and CM $(p=0,02$ and $p=0,007$, respectively); IL- I0 was significantly higher in UM $(p=0,0009), S M(p=0,000 I)$ and CM $(p=0,000 I)$ 
Table 4: Cytokine distribution in the Indian population: median TNF, IFN- $\gamma$ and IL-I 0 per clinical group.

\begin{tabular}{|c|c|c|c|c|c|c|}
\hline & NEC (min-max) & EC (min-max) & UM (min-max) & SM (min-max) & CM (min-max) & $\begin{array}{c}\mathrm{ExCM} \text { (min- } \\
\max )\end{array}$ \\
\hline TNF (pg/ml) & $57(39-73)$ & $78(63-120)$ & 180 (1 I9-207) & $200(|73-38|)$ & $530(258-1227)$ & $81(69-124)$ \\
\hline IFN- $\gamma(\mathbf{p g} / \mathrm{ml})$ & $23(|0-3|)$ & $22(\mid 7-201)$ & $119(70-153)$ & $127(|| \mid-200)$ & $65(45-101)$ & $32(\mathrm{II}-53)$ \\
\hline IL-I 0 (pg/ml) & $13(5-26)$ & $22(||-3 \mid)$ & $120(97-147)$ & $176(12 \mid-253)$ & $301(175-506)$ & $85(40-108)$ \\
\hline IL-4 (pg/ml) & $18(8-40)$ & $58(23-84)$ & $65(31-204)$ & $69(50-89)$ & $62(40-85)$ & $48(29-80)$ \\
\hline
\end{tabular}

NEC - non endemic control, EC - endemic control, UM - uncomplicated malaria, SM - severe malaria (non-cerebral), CM - cerebral malaria, ExCM - Ex-cerebral malaria.

Comparing group by group with the Endemic control: TNF was significantly lower in NEC $(p=0,0006)$ and higher in UM $(p=1,19 \times 10-7), \operatorname{SM}(p$

$=1,01 \times 10-5)$ and $C M(p=2,26 \times 10-7)$; IFN- $\gamma$ was significantly higher in all diseased groups $(U M-p=5, I I \times 10-6, S M-p=1,01 \times 10-6, C M-P$

$=9,7 \mathrm{I} \times 10-6)$; IL-10 was significantly higher in all diseased groups $(\mathrm{UM}-\mathrm{p}=1,04 \times 10-7, \mathrm{SM}-\mathrm{p}=1,0 \mathrm{I} \times 10-5$ and $C M-\mathrm{p}=2,46 \times 10-7)$.

levels are higher in UM and SM (Figure 1C and 1D) than in CM patients who had values similar to that of the controls $[8,24]$. In addition, the median levels of circulating IgE in the ExCM group were close to that of the CM group. Also, no significant correlation was found between both IgE and TNF levels in the CM group. These observations are contrary to published data describing increased IgE levels that correlate with high concentrations of circulating TNF, a cytokine associated with malaria severity and also with pRBC adherence on brain capillary endothelial cells $[9,10,25,26]$. The results suggest that either IgE does not play an important role in CM pathogenesis, or that these antibodies may participate in the parasite sequestration into the brain or other organ capillaries [24].

There was a significant increase in IgE levels with age in Gabonese children independent of the disease group (Figure 2). This increase in IgE production between 0 and six years of age may also reflect an increase in the capacity of the immune system to respond to parasite infections $[7,27]$. Such a correlation was not found in Indian groups. This would be expected because the Indian groups comprised mainly adults, with the few children being older than five years. Although most of individuals in the Gabonese endemic control group had already been in contact with the parasite, as demonstrated by the high titres of specific antibody to $P$. falciparum-infected red blood cells observed in these children, the median plasma IgE concentrations were compared with those of the Indian NEC group. Although the median total IgE levels in the Indian NEC groups was higher than that of the Gabonese EC group, suggesting that age could be an important factor in IgE production, the higher IgE levels in the Indian population may also be interpreted as the result of either environmental factors, such as predominance of food allergies, of the genetic background, which may predispose to developing IgE responses $[13,27,29$ 32] or of a co-infection with other parasites, such as helminths affecting the IgE responses in these patients $[33,34]$. In the Gabonese cohorts, IgE levels tended to cor- relate negatively to parasitaemia except in UM whereas all patient groups from India showed a positive correlation.

The pool of circulating IgE comprises both monomeric and complexed immunoglobulins [35,36]. A functional test was performed, based on the ability of the circulating IgE from the sera of different patient groups to induce degranulation of mast cells in the presence of $\mathrm{pRBC}$ antigens to better estimate the $P$. falciparum specific IgE response. This test does not provide specific IgE concentration within total IgE. It is based on the specific IgE induced percentage of mast cells degranulation. Degranulation was measured by quantifying $\beta$-hexosaminidase release. Functional $P$. falciparum-specific IgEs were detected in randomly chosen patients from all groups in both the Gabonese and Indian populations, except for ExCM Indian patients. The highest percentage of patients with functional anti-parasite IgEs was found in the Gabonese AI and UM and Indian EC and UM groups, which decreased in the SM and CM groups (Figure 3A and $3 B$ ). This suggests a protective role for $P$. falciparum-specific IgE, and is consistent with previous published data $[13,14]$.

Although the CM group had a low percentage of patients able to induce degranulation, it was the only group where there was one patient serum inducing a mast cell degranulation above $30 \%$. This intense response may be associated with the presence of IgE with higher affinity for $P$. falciparum antigens, as previously reported by GonzalezEspinosa et al. [37]. However, the level of degranulation can also be enhanced by the number of receptors involved in recognizing the antigen-IgE complex, which can strongly affect the size of the secretory response $[37,38]$. There was no evident correlation between the level of functional $P$. falciparum-specific IgE (percentage of enzyme release) and the level of total IgE per group within each population. This is unsurprising, given that both the monomeric and complexed forms of circulating parasitespecific IgE can affect the level of degranulation [39-41]. It 


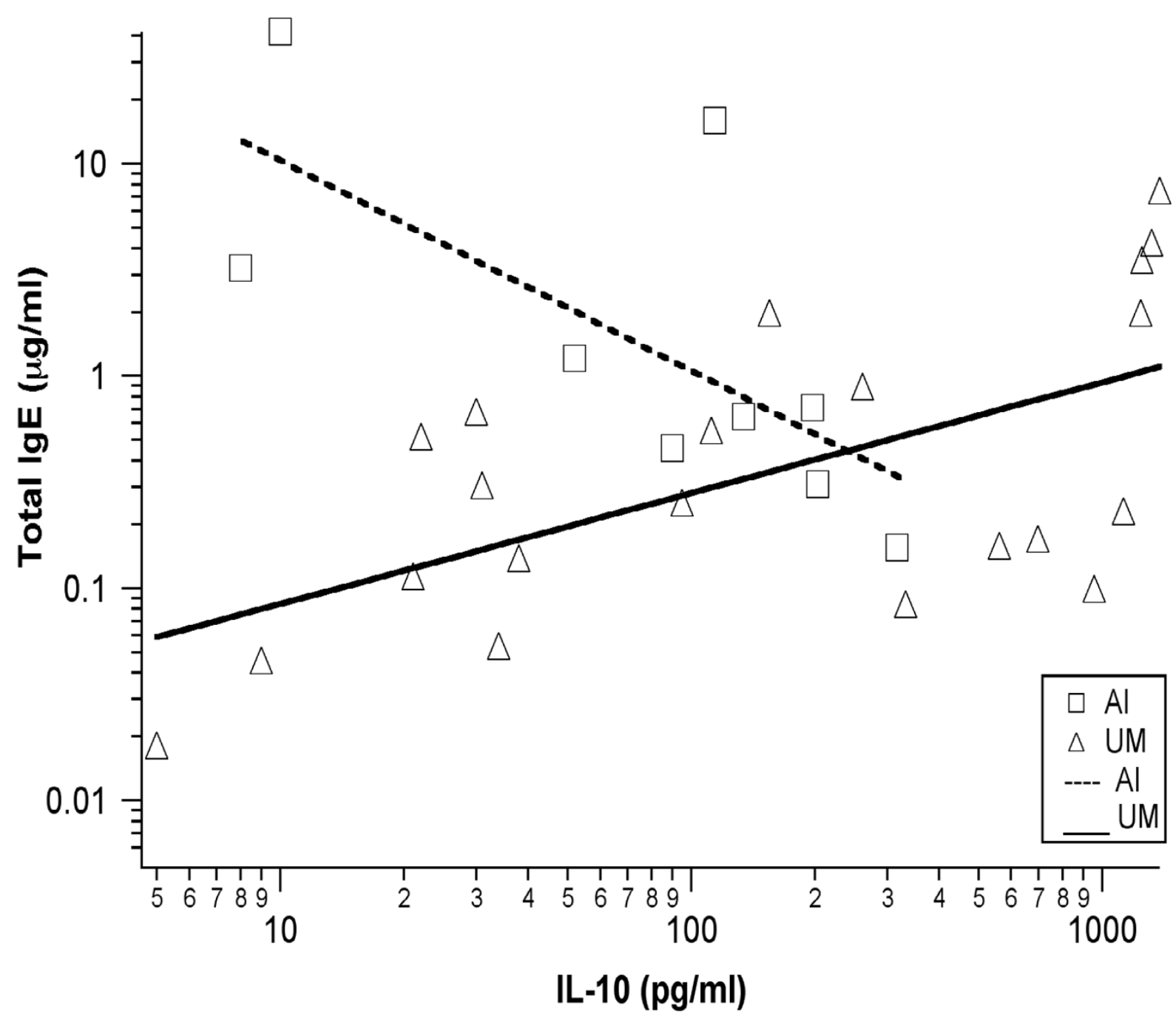

Figure 4

IL-10 correlation with total IgE levels in the Gabonese population. Dashed line - Asymptomatic patients (significant negative spearman correlation, $p=0.025$ ). Bold line - Uncomplicated malaria patients (significant positive correlation, $p=0.017$ ).

is also acceptable that total IgE levels were not directly correlated to FcåRI upregulation levels [40,42]. The minimum level of receptors occupied by the parasite antigenspecific IgE complex required to induce a degranulation response is $10 \%$. Consequently, this response will be independent of the total IgE levels [20]. An increase in specific IgE levels has been also seen in other parasitic infections, such as helminthiasis, in which the specific IgEs usually help to eliminate the pathogens either through hypersensitivity reactions resulting from mast cell degranulation or by inducing antibody-dependent cell-mediated responses [27].
High levels of IgE in P. falciparum-infected individuals have been shown to be due to an underlying imbalance in favour of IL- 4 in the ratio of CD4+ T cell producers, which are responsible for the IgG/IgM isotype switching to IgE [10]. High levels of circulating IL-4 have also been associated with a greater parasite antigen-specific production of IgE in individuals less susceptible to malaria [13]. Also, Th1-type pro-inflammatory cytokines, such as IFN- $\gamma$ and TNF are thought to play an important role in the both protecting against and increasing the pathogenesis of cerebral malaria $[3,4,22]$. Plasma TNF, IFN- $\gamma$ and IL-10 levels were measured in both the Indian and Gabonese groups. In the 

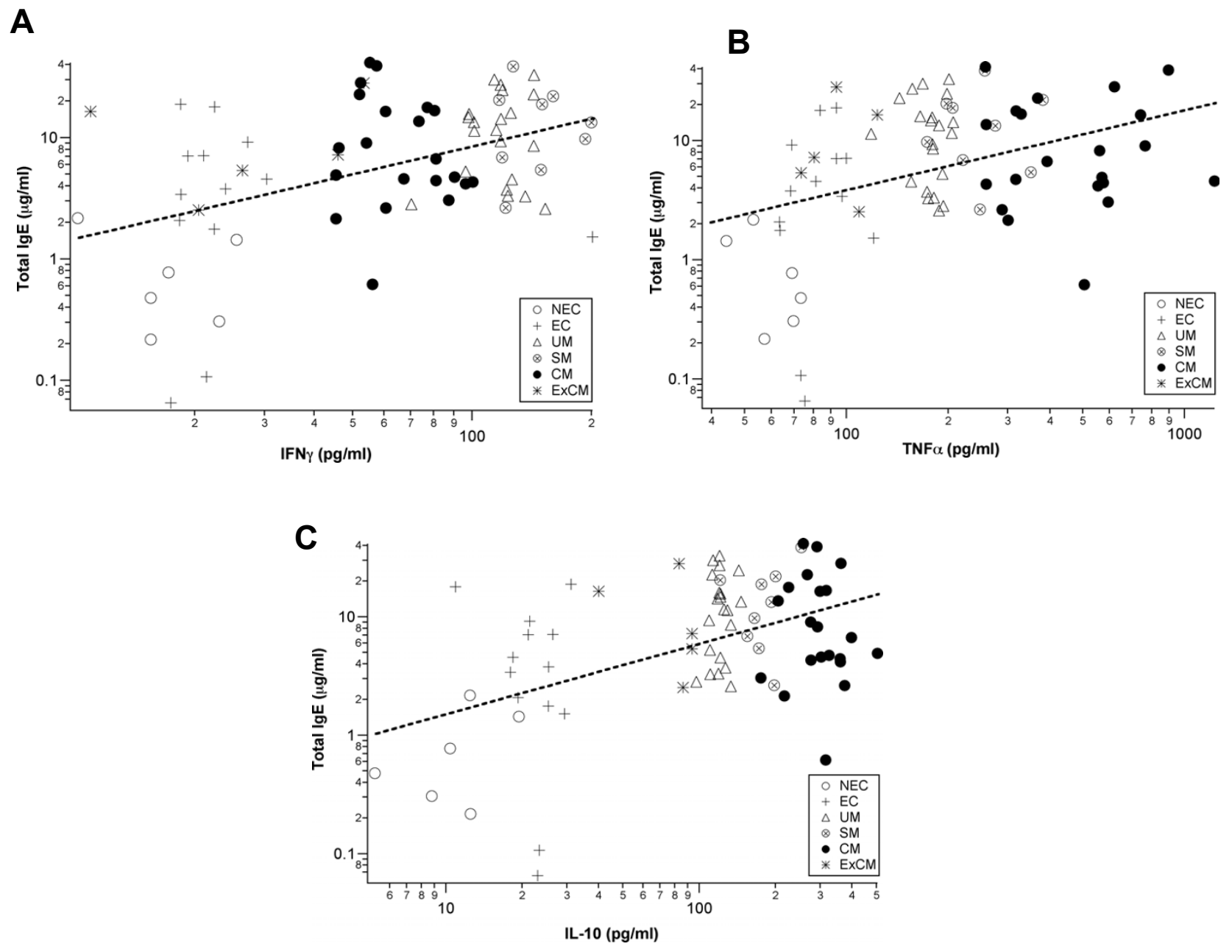

\section{Figure 5}

Cytokine correlation with lgE levels in the Indian population. A. TNF correlation with total lgE levels (significant positive spearman correlation, $p=0.0037$ ); B. IFN- $\gamma$ correlation with total $\lg E$ levels (Significant positive spearman correlation, $p=0.0028$ ); C. IL-IO correlation with total IgE levels (significant positive spearman correlation, $P=0.005 \mathrm{I}$ ).

Gabonese groups, no direct correlation was found between IgE and IFN- $\gamma$ or TNF levels in the UM, SM and $\mathrm{CM}$ patients. However, the level of IgE production was correlated to IL-10 levels in UM and AI patients (Figure 4). In this group, there was a positive association between IL10 levels and parasite load, but not between IgE levels and parasitaemia. In the Indian groups, IFN- $\gamma$, TNF and IL-10 levels were all significantly correlated with IgE levels independent of the group (Figures 5A, B and 5C). When looking for a correlation per group, no significant statistic value was found. IL-4 is the main cytokine responsible for IgE production, inducing antibody isotype switching from IgG and IgM to IgE [10]. Previous studies have shown an association between the IL-4/IFN- $\gamma$ levels and IgE levels, suggesting an induced Th2-type switched response [43]. As IL-4 levels were different between groups only in Indians, a correlation was expected between this ratio and IgE levels, and surprisingly, the results showed an opposite correlation $(\mathrm{p}=0,012)$. The levels of IL-4 remained almost constant across all groups as IgE levels increased. Therefore, this opposite correlation arises due to a parallel increase in both IFN- $\gamma$ and IgE levels. This suggests that, in the Indian population, IL- 4 does not seem to directly influence IgE levels. It has been shown experimentally that there are conditions under which alternative mechanisms may induce IgE production independent of IL-4 [44]. Regarding other cytokine ratios, like TNF/IL-10, no significant correlation was found with total IgE levels. 
In conclusion, these results showed that total IgE levels increased in infected patients, mainly in UM and SM patients but not in CM patients. The decrease in total IgE levels in the CM group was associated with a higher intensity of mast cell degranulation induced in vitro by $P$. falciparum-specific IgE. In addition, no correlation was found between total and functional $P$. falciparum-specific IgE levels.

The results reported here show the high activity of functional circulating $P$. falciparum-specific IgE in asymptomatic malaria patients. However, no correlation was observed between plasma levels of total IgE, the disease phenotype and the cytokines pattern in the different groups of patients studied. The opposite association between pro- and anti-inflammatory cytokine ratios and IgE levels reveals the complexity of immune response disruption occurring during malaria in patients from low and high malaria endemic region.

\section{Authors' contributions}

Joana Duarte contributed to the acquisition, analysis, interpretation of data and manuscript drafting but not the design of the study. Prakash Deshpande and Vincent Guiyedi have highly contributed to conception and interpretation of data and manuscript revising. Salah Mécheri collaborated to the design and interpretation of IgE functional tests and manuscript drafting. Constantin Fesel contributed to the statistical analysis, interpretation of data and manuscript drafting.

Pierre-André Cazenave, Gyan C. Mishra and Maryvonne Kombila have contributed to conception of the study and manuscript revising. Sylviane Pied participated to conception, design, analysis and interpretation of data, the final approval of the version to be published.

\section{Financial support}

This work was part of the Centre National de la Recherche Scientifique-Laboratoires Européens Associés "Génétique et developpement de la tolérance naturelle" program. It was supported by the PAL+ program of the French Ministry of Research. We thank the Indo-French Centre for the Promotion of Advanced Research (IFCPAR), New Delhi, India for providing financial assistance (project No.21033). C-F received a post-doctoral fellowship from the Fundaçâo para a Ciência e Tecnologia (Portugal). V-G holds a fellowship from the Agence Universitaire de la Francophonie (AUF).

\section{Acknowledgements}

We thank M. Bouyou-Akotet and M. Idrissa-Boubou for assistance with the collection of plasma samples. We are grateful to R.O. for critical reading of and his help in preparing the manuscript.

\section{References}

I. Moorthy VS, Good MF, Hill AV: Malaria vaccine developments. Lancet 2004, 363:I50-156.

2. Miller LH, Baruch DI, Marsh K, Doumbo OK: The pathogenic basis of malaria. Nature 2002, 4I 5:673-679.

3. Heddini A: Malaria pathogenesis: a jigsaw with an increasing number of pieces. Int J Parasitol 2002, 32:1587-1598.

4. de Souza JB, Riley EM: Cerebral malaria: the contribution of studies in animal models to our understanding of immunopathogenesis. Microbes Infect 2002, 4:291-300.

5. Adams S, Brown H, Turner G: Breaking down the blood-brain barrier: signaling a path to cerebral malaria? Trends Parasitol 2002, 18:360-366.

6. Miller LH, Good MF, Milon G: Malaria pathogenesis. Science 1994, 264: $1878-1883$.

7. Calissano C, Modiano D, Sirima BS, Konate A, Sanou I, Sawadogo A, Perlmann H, Troye-Blomberg M, Perlmann P: IgE antibodies to $P$. falciparum and severity of malaria in children of one ethnic group living in Burkina Faso. Am J Trop Med Hyg 2003, 69:3I-35.

8. Perlmann H, Helmby H, Hagstedt M, Carlson J, Larsson PH, TroyeBlomberg M, Perlmann P: IgE elevation and IgE anti-malarial antibodies in $P$. falciparum malaria: association of high IgE levels with cerebral malaria. Clin Exp Immunol 1994, 97:284-292.

9. Perlmann P, Perlmann H, ElGhazali G, Blomberg MT: IgE and tumor necrosis factor in malaria infection. Immunol Lett 1999, 65:29-33.

10. Perlmann P, Perlmann H, Flyg BW, Hagstedt M, Elghazali G, Worku S, Fernandez V, Rutta AS, Troye-Blomberg M: Immunoglobulin E, a pathogenic factor in P. falciparum malaria. Infect Immun 1997, 65: $116-12 \mid$.

II. Perlmann P, Perlmann H, Looareesuwan S, Krudsood S, Kano S, Matsumoto Y, Brittenham G, Troye-Blomberg M, Aikawa M: Contrasting functions of IgG and IgE antimalarial antibodies in uncomplicated and severe $\boldsymbol{P}$. falciparum malaria. Am J Trop Med Hyg 2000, 62:373-377.

12. Seka-Seka J, Brouh Y, Yapo-Crezoit AC, Atseye NH: The role of serum immunoglobulin $E$ in the pathogenesis of $P$. falciparum malaria in Ivorian children. Scand J Immunol 2004, 59:228-230.

13. Bereczky S, Montgomery SM, Troye-Blomberg M, Rooth I, Shaw MA, Farnert A: Elevated anti-malarial IgE in asymptomatic individuals is associated with reduced risk for subsequent clinical malaria. Int J Parasitol 2004, 34:935-942.

14. Farouk SE, Dolo A, Bereczky S, Kouriba B, Maiga B, Farnert A, Perlmann H, Hayano M, Montgomery SM, Doumbo OK, Troye-Blomberg M: Different antibody- and cytokine-mediated responses to $P$. falciparum parasite in two sympatric ethnic tribes living in Mali. Microbes Infect 2005, 7:1 I0-117.

15. Oettgen HC, Geha RS: IgE regulation and roles in asthma pathogenesis. J Allergy Clin Immunol 200I, 107:429-440.

16. Nyakeriga MA, Troye-Blomberg M, Bereczky S, Perlmann H, Perlmann P, EIGhazali G: Immunoglobulin E (IgE) containing complexes induce IL-4 production in human basophils: effect on Thl-Th2 balance in malaria. Acta Trop 2003, 86:55-62.

17. Reischl IG, Coward WR, Church MK: Molecular consequences of human mast cell activation following immunoglobulin Ehigh-affinity immunoglobulin E receptor (IgE-FcepsilonRI) interaction. Biochem Pharmacol 1999, 58:1841-1850.

18. WHO: Severe and complicated malaria. Trans $R$ Soc Trop Med Hyg 1990, 84: I-65.

19. Trager W, Jensen JB: Human malaria parasites in continuous culture. Science 1976, 193:673-675.

20. Marchand F, Mecheri S, Guilloux L, lannascoli B, Weyer A, Blank U: Human serum IgE-mediated mast cell degranulation shows poor correlation to allergen-specific IgE content. Allergy 2003, 58:1037-1043.

21. Takagi K, Nakamura R, Teshima R, Sawada J: Application of human Fc epsilon RI alpha-chain-transfected RBL-2H3 cells for estimation of active serum IgE. Biol Pharm Bull 2003, 26:252-255.

22. Plebanski M, Hill AV: The immunology of malaria infection. Curr Opin Immunol 2000, I 2:437-44I.

23. Torre D, Speranza F, Giola M, Matteelli A, Tambini R, Biondi G: Role of ThI and Th2 cytokines in immune response to uncomplicated P. falciparum malaria. Clin Diagn Lab Immunol 2002, 9:348-35I.

24. Maeno Y, Perlmann P, PerImann H, Kusuhara Y, Taniguchi K, Nakabayashi $\mathrm{T}$, Win K, Looareesuwan S, Aikawa M: IgE deposition in 
brain microvessels and on parasitized erythrocytes from cerebral malaria patients. Am J Trop Med Hyg 2000, 63: I28-I32.

25. Artavanis-Tsakonas K, Tongren JE, Riley EM: The war between the malaria parasite and the immune system: immunity, immunoregulation and immunopathology. Clin Exp Immunol 2003, 133:145-152.

26. Chen $\mathrm{Q}$, Schlichtherle $\mathrm{M}$, Wahlgren $\mathrm{M}$ : Molecular aspects of severe malaria. Clin Microbiol Rev 2000, 13:439-450.

27. Winter WE, Hardt NS, Fuhrman S: Immunoglobulin E: importance in parasitic infections and hypersensitivity responses. Arch Pathol Lab Med 2000, I 24: I 382-I 385.

28. Migot-Nabias F, Mombo LE, Luty AJ, Dubois B, Nabias R, Bisseye C, Millet $\mathrm{P}$, $\mathrm{Lu} \mathrm{CY}$, Deloron $\mathrm{P}$ : Human genetic factors related to susceptibility to mild malaria in Gabon. Genes Immun 2000, I:435-44I.

29. Poulsen LK: Allergy assessment of foods or ingredients derived from biotechnology, gene-modified organisms, or novel foods. Mol Nutr Food Res 2004, 48:413-423.

30. Yazdanbakhsh M, Kremsner PG, van Ree R: Allergy, parasites, and the hygiene hypothesis. Science 2002, 296:490-494.

31. Paganotti GM, Babiker HA, Modiano D, Sirima BS, Verra F, Konate A, Ouedraogo AL, Diarra A, Mackinnon MJ, Coluzzi M, Walliker D: Genetic complexity of $P$. falciparum in two ethnic groups of Burkina Faso with marked differences in susceptibility to malaria. Am J Trop Med Hyg 2004, 71: 173-178.

32. Verra F, Luoni G, Calissano C, Troye-Blomberg M, Perlmann P, Perlmann $H$, Arca B, Sirima BS, Konate A, Coluzzi M, Kwiatkowski D, Modiano D: IL4-589C/T polymorphism and IgE levels in severe malaria. Acta Trop 2004, 90:205-209.

33. Nacher M, Gay F, Singhasivanon P, Krudsood S, Treeprasertsuk S, Mazier D, Vouldoukis I, Looareesuwan S: Ascaris lumbricoides infection is associated with protection from cerebral malaria. Parasite Immunol 2000, 22: I07-I I 3.

34. Nacher M, Singhasivanon P, Traore B, Vannaphan S, Gay F, Chindanond D, Franetich JF, Mazier D, Looareesuwan S: Helminth infections are associated with protection from cerebral malaria and increased nitrogen derivatives concentrations in Thailand. Am J Trop Med Hyg 2002, 66:304-309.

35. Johansson SG: Anti-IgE antibodies in human serum. J Allergy Clin Immunol 1986, 77:555-557.

36. Marone G, Spadaro G, Palumbo C, Condorelli G: The anti-IgE/antiFcepsilonRlalpha autoantibody network in allergic and autoimmune diseases. Clin Exp Allergy 1999, 29:17-27.

37. Gonzalez-Espinosa C, Odom S, Olivera A, Hobson JP, Martinez ME, Oliveira-Dos-Santos A, Barra L, Spiegel S, Penninger JM, Rivera J: Preferential signaling and induction of allergy-promoting lymphokines upon weak stimulation of the high affinity IgE receptor on mast cells. J Exp Med 2003, 197: |453- I 465.

38. Posner RG, Paar JM, Licht A, Pecht I, Conrad DH, Hlavacek WS: Interaction of a monoclonal IgE-specific antibody with cellsurface IgE-Fc epsilon RI: characterization of equilibrium binding and secretory response. Biochemistry 2004, 43:11352-11360.

39. Kalesnikoff J, Huber M, Lam V, Damen JE, Zhang J, Siraganian RP, Krystal G: Monomeric IgE stimulates signaling pathways in mast cells that lead to cytokine production and cell survival. Immunity 200I, I 4:80I-8II.

40. Kitaura J, Xiao W, Maeda-Yamamoto M, Kawakami Y, Lowell CA, Kawakami T: Early divergence of Fc epsilon receptor I signals for receptor up-regulation and internalization from degranulation, cytokine production, and survival. I Immunol 2004, 173:4317-4323.

41. Yamaguchi M, Sayama K, Yano K, Lantz CS, Noben-Trauth N, Ra C, Costa J], Galli SJ: IgE enhances Fc epsilon receptor I expression and IgE-dependent release of histamine and lipid mediators from human umbilical cord blood-derived mast cells: synergistic effect of IL-4 and IgE on human mast cell Fc epsilon receptor I expression and mediator release. J Immunol 1999, 162:5455-5465.

42. Saini SS, Klion AD, Holland SM, Hamilton RG, Bochner BS, Macglashan DW: The relationship between serum IgE and surface levels of FcepsilonR on human leukocytes in various diseases: correlation of expression with FcepsilonRI on basophils but not on monocytes or eosinophils. J Allergy Clin Immunol 2000, 106:514-520.
43. Elghazali G, Perlmann H, Rutta AS, Perlmann P, Troye-Blomberg M: Elevated plasma levels of IgE in $P$. falciparum -primed individuals reflect an increased ratio of IL-4 to interferon-gamma (IFN-gamma)-producing cells. Clin Exp Immunol 1997 , 109:84-89.

44. Grunewald SM, Teufel M, Erb K, Nelde A, Mohrs M, Brombacher F, Brocker EB, Sebald $W$, Duschl A: Upon prolonged allergen exposure IL-4 and IL-4Ralpha knockout mice produce specific IgE leading to anaphylaxis. Int Arch Allergy 200I, I 25:322-328.
Publish with Bio Med Central and every scientist can read your work free of charge

"BioMed Central will be the most significant development for disseminating the results of biomedical research in our lifetime. "

Sir Paul Nurse, Cancer Research UK

Your research papers will be:

- available free of charge to the entire biomedical community

- peer reviewed and published immediately upon acceptance

- cited in PubMed and archived on PubMed Central

- yours - you keep the copyright

Submit your manuscript here:

http://www.biomedcentral.com/info/publishing_adv.asp
BioMedcentral 